Chapman University

Chapman University Digital Commons

Pharmacy Faculty Articles and Research

School of Pharmacy

7-8-2008

\title{
Surface Charge Effects Involved in the Control of Stability of Sols Comprising Uniform Cholesterol Particles
}

Vuk Uskoković

Chapman University, uskokovi@chapman.edu

Follow this and additional works at: http://digitalcommons.chapman.edu/pharmacy_articles

Part of the Cardiovascular Diseases Commons, Medical Biochemistry Commons, and the Other Chemistry Commons

\section{Recommended Citation}

Uskoković V. Surface charge effects involved in the control of stability of sols comprising uniform cholesterol particles. Materials and Manufacturing Processes. 2008;23(6):620-623. doi: 10.1080/10426910802160692

This Article is brought to you for free and open access by the School of Pharmacy at Chapman University Digital Commons. It has been accepted for inclusion in Pharmacy Faculty Articles and Research by an authorized administrator of Chapman University Digital Commons. For more information, please contact laughtin@chapman.edu. 


\section{Surface Charge Effects Involved in the Control of Stability of Sols Comprising Uniform Cholesterol Particles}

\section{Comments}

This is an Accepted Manuscript of an article published in Materials and Manufacturing Processes, volume 23, issue 6, in 2008, available online: http:/ / www.tandfonline.com/doi/abs/10.1080/10426910802160692.

\section{Copyright}

Taylor \& Francis 


\title{
Surface Charge Effects Involved in the Control of Stability of
}

\section{Sols Comprising Uniform Cholesterol Particles}

\author{
Vuk Uskoković ${ }^{*}$ \\ Center for Advanced Materials Processing, Clarkson University, Potsdam, NY 13699-5814, USA
}

\begin{abstract}
The reported is a method for the preparation of stable dispersions of narrowly dispersed cholesterol crystals, which may provide an excellent model for the aggregation studies thereof. The effects of experimental parameters that include concentration, $\mathrm{pH}$, ionic strength, temperature and aging on the resulting morphologies are acknowledged. Regardless of the cause of destabilization, the multilayered aggregates of cholesterol particles are formed by "face-to-face" stacking of the original platelets, as promoted by higher surface charge density on particle sides comparing to their faces. In accordance with the crucial involvement of surface charge effects in stabilization of cholesterol dispersions produced hereby, aggregation of the original particles becomes enhanced at increased salt contents and at $\mathrm{pH}$ conditions that correspond to the isoelectric point of the dispersed particles.
\end{abstract}

Keywords: Cholesterol, Sol, Surface Charge, Uniform particles, Zeta potential.

\section{Introduction}

As the most abundant steroid in the animal kingdom, involved in sustaining cell membranes and acting as a precursor for the biological synthesis of bile acids, vitamin $\mathrm{D}_{3}$ and steroid hormones, cholesterol presents an essential biomolecule for human body

\footnotetext{
${ }^{*}$ Currently at: Division of Biomaterials and Bioengineering, University of California, 513 Parnassus Avenue, San Francisco, CA 94143, USA
} 
functioning ${ }^{1}$. However, due to unbalanced relationship between the internal level of cholesterol in the body and the action of lipid bilayers and vesicles in bile and lipoprotein complexes in blood that regulate its solubilization, cholesterol may precipitate forming gallstones, kidney stones and atherosclerotic deposits, thus endangering health of individual organisms.

Yet even when depositing along body fluid pathways as a result of pathological states of the organism, nature has evolved mechanisms for minimizing the damage. Namely, cholesterol molecules crystallize in double-layer structures with end-for-end arrangement of approximately parallel molecules ${ }^{2,3}$ (Fig.1). The growth of cholesterol crystals into typical platelet forms results from a faster growth within the bilayer plane comparing to the growth between bilayers, initiated by stronger interactions between neighboring molecules in the same bilayer plane ${ }^{4}$. Such form of deposition provides maximization of the buildup of cholesterol coagulates in arteries, gallbladder and intestinal lumen without constraining the natural flow of life-sustaining fluids. The dissolution of cholesterol in form of layers, instead of crumbles, may be considered as another consequence of the biomolecular co-evolution of cholesterol and multicellular organisms ${ }^{5}$. On the other hand, relatively high surface energy of plate-shaped crystals favors their adhesion on membranes and walls of the respective organs and tissues, decreasing their susceptibility towards efficient dissolution and removal. Understanding the physicochemical pathways involved in cholesterol precipitation and deposition processes correspondingly presents one of the important contemporary challenges that may provide us with far-stretching potential benefits. 


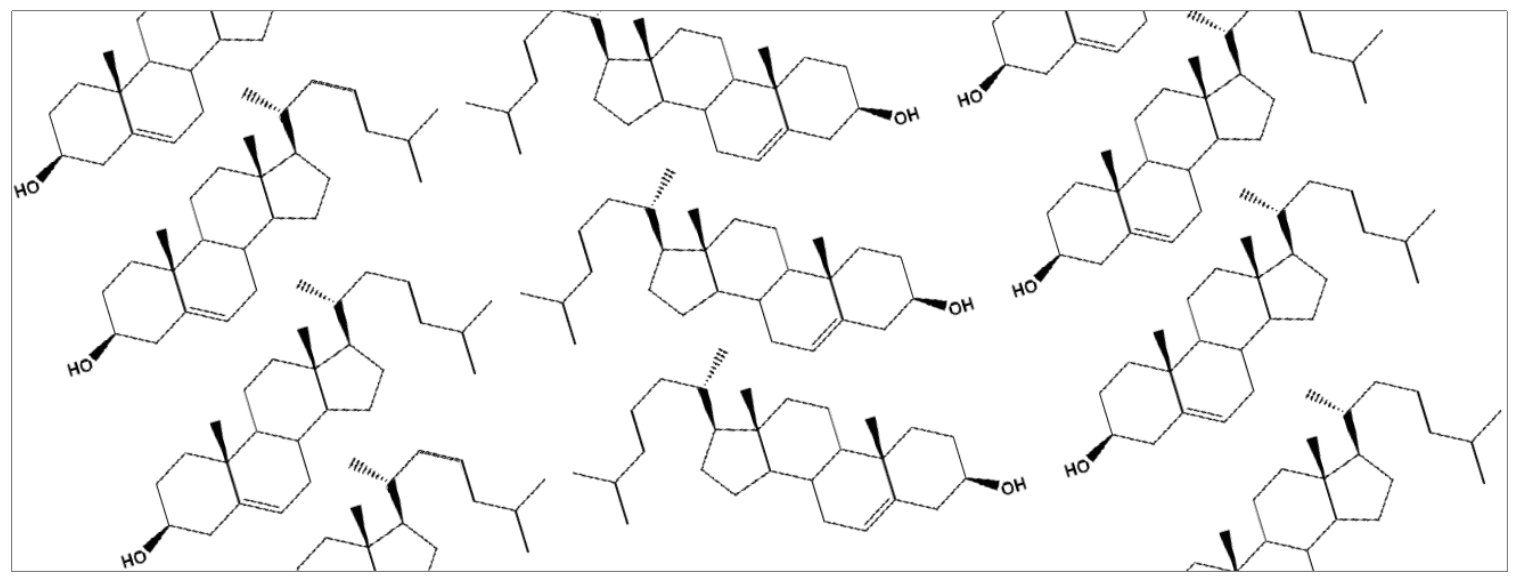

Fig.1. In-plane end-for-end arrangement of cholesterol molecules in their crystalline form.

This study appears to be the first to describe the preparation of uniform colloidal crystallites of cholesterol, to characterize some of their properties, and to examine the propensity of the corresponding singlets to form multilayered aggregates ${ }^{6}$. The dispersions obtained hereby can serve as viable model systems for the study of the effects of various physical and chemical environments on the formation, stability and interactions of cholesterol crystals with different solutes and substrates ${ }^{7}$. As another part of the actual study, the specific interaction between cholesterol and a water-soluble derivative of cellulose has been shown to inhibit the tendency of solid cholesterol to adopt typical plate- or needle- shaped morphologies ${ }^{8}$. Also, crystallization of cholesterol in chemical conditions that involved the presence of emulsions composed of surface active agents of different ionic nature has indicated that spherical "templates" of fine emulsion droplets may impose spherical morphologies to cholesterol particles nucleated within $^{9}$. 


\section{Experimental part}

To obtain the stable dispersions of uniform cholesterol particles, $15 \mathrm{ml}$ of water is abruptly poured into the solution comprising $20 \mathrm{mg}$ of cholesterol dissolved in $10 \mathrm{ml}$ of 1-propanol. After $10 \mathrm{~min}$ of aging the non-agitated aqueous-alcoholic sol at atmospheric conditions, a few drops thereof are deposited onto a sample carrier for Field-Emission Scanning Electron Microscopy (JEOL JSM-7400F), dried in air and analyzed. Crystallinity of the cholesterol powders was examined with a Bruker D8 Axis X-ray diffractometer, whereas electrophoretic measurements were performed using a BIC ZetaPlus Analyzer.

\section{Results and discussion}

Precipitation of cholesterol from simple solutions has been on one hand evidenced as a complex process interdependent on many experimental variables, whereas on the other hand the tendency of cholesterol molecules to adopt layered crystalline forms, manifested as various platelet morphologies, has dominated the precipitation processes. X-ray diffraction pattern of the obtained uniform cholesterol particles is shown in Fig.2. Changes in solvent identity and composition, cholesterol concentration, $\mathrm{pH}$, ionic strength, temperature and aging treatment all affect the resulting morphologies in specific ways. The most satisfying uniformity of the prepared cholesterol particles was observed with using 1-propanol as a solvent and water as a precipitation agent (Fig.3a). Monodisperse platelets were formed within a narrow range of experimental conditions, 
whereas mild deviations from the optimal cholesterol concentration or solvent/nonsolvent ratio led to disruption of well-defined and narrowly dispersed character of individual particles and the stability of the corresponding suspensions. Whereas rapid nucleation of the solid phase may lead to the formation of monodisperse platelets with 1$2 \mu \mathrm{m}$ in size, evaporation-induced nucleation results in the formation of millimeter-sized deposits. Broader size distributions were typically observed as the result of decreased nucleation rate.

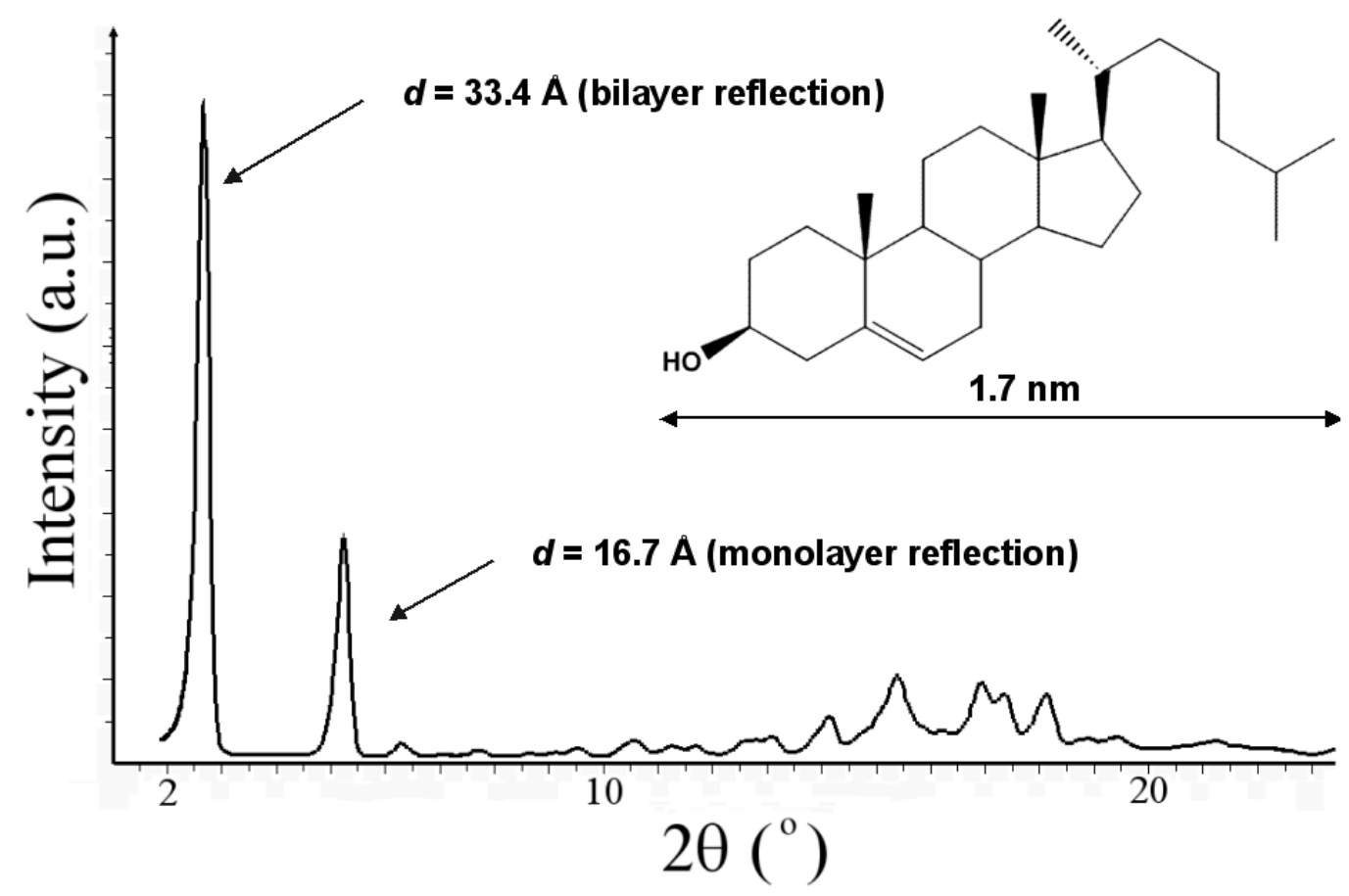

Fig.2. X-ray diffraction pattern of the prepared uniform cholesterol particles. The most intensive peak at $2 \theta$ $=2.6^{\circ}$ corresponds to the Bragg distance of $d=33.4 \AA$ and, henceforth, to constructive interference of reflections from cholesterol bilayers, whereas the peak at $2 \theta=5.2^{\circ}$ corresponds to the Bragg distance of $d$ $=16.7 \AA$ and, therefore, to constructive interference of reflections from cholesterol monolayers.

Aging of the suspensions at first leads to the thickening of initially thin leaflet particles into brick-shaped ones. After this, diffusion-driven growth stage, the 
aggregation of platelets takes place until multilayered cholesterol cuboids with refined edges are formed (Fig.3b). The fact that hydrogen bonds and van der Waals forces figure as major intermolecular bonds within and between bilayers, respectively, and are yet engaged in particle-particle interactions explains the smoothness and the lack of porosity of the final aggregates. Aging at increased temperatures leads to an increased level of sintering of individual particles that eventually gives rise to large cholesterol blocks, which although maintain their typical rectangular morphological character (Fig.3c). The effect of dissolution of particles due to temperature-induced increases in solubility and the effect of their aggregation due to increased level of thermal movement compete and exactly at the body-temperature $\left(37^{\circ} \mathrm{C}\right)$ produce the most optimal conditions for comparatively fast aggregation and sintering of the particles.
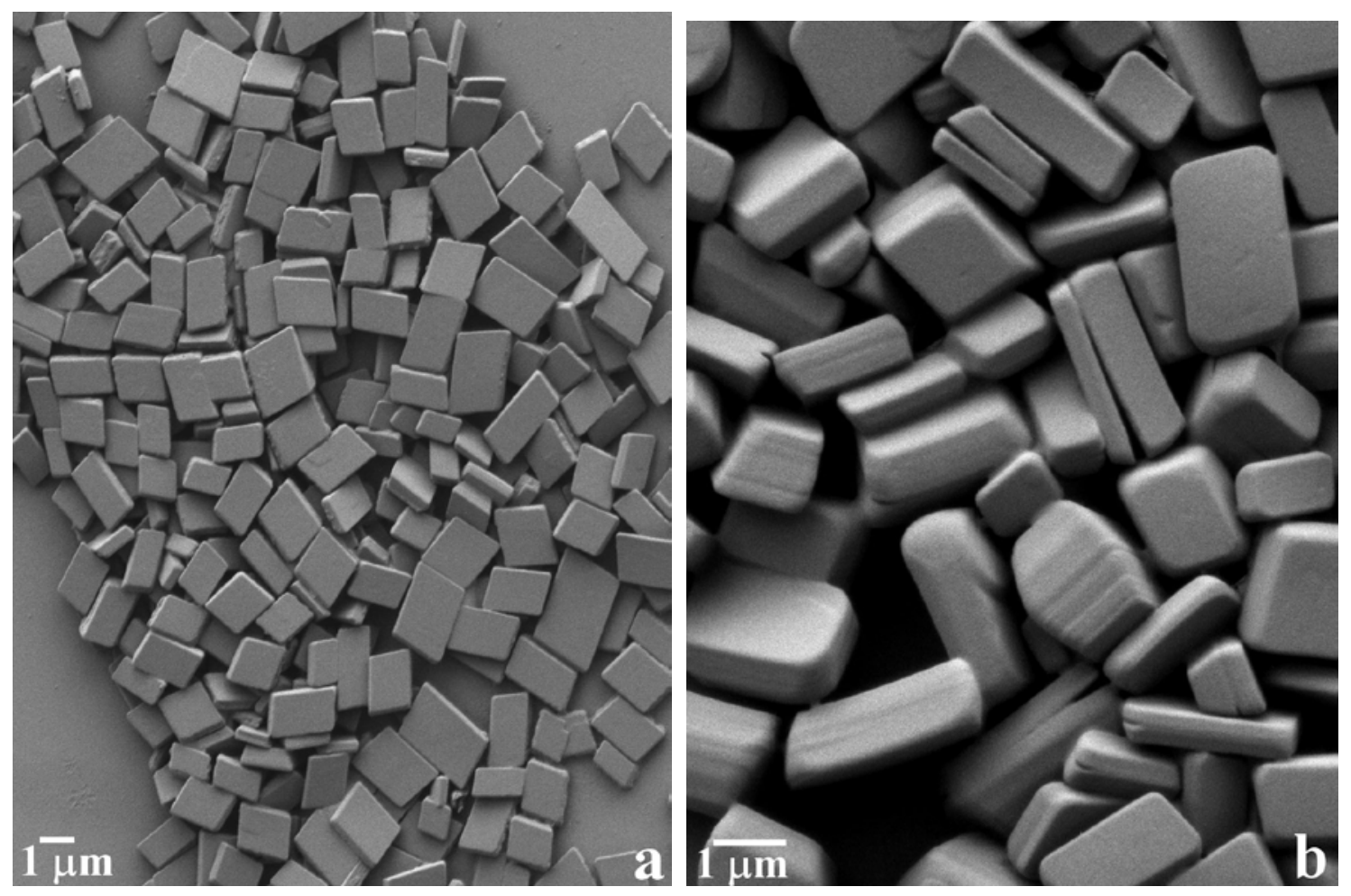

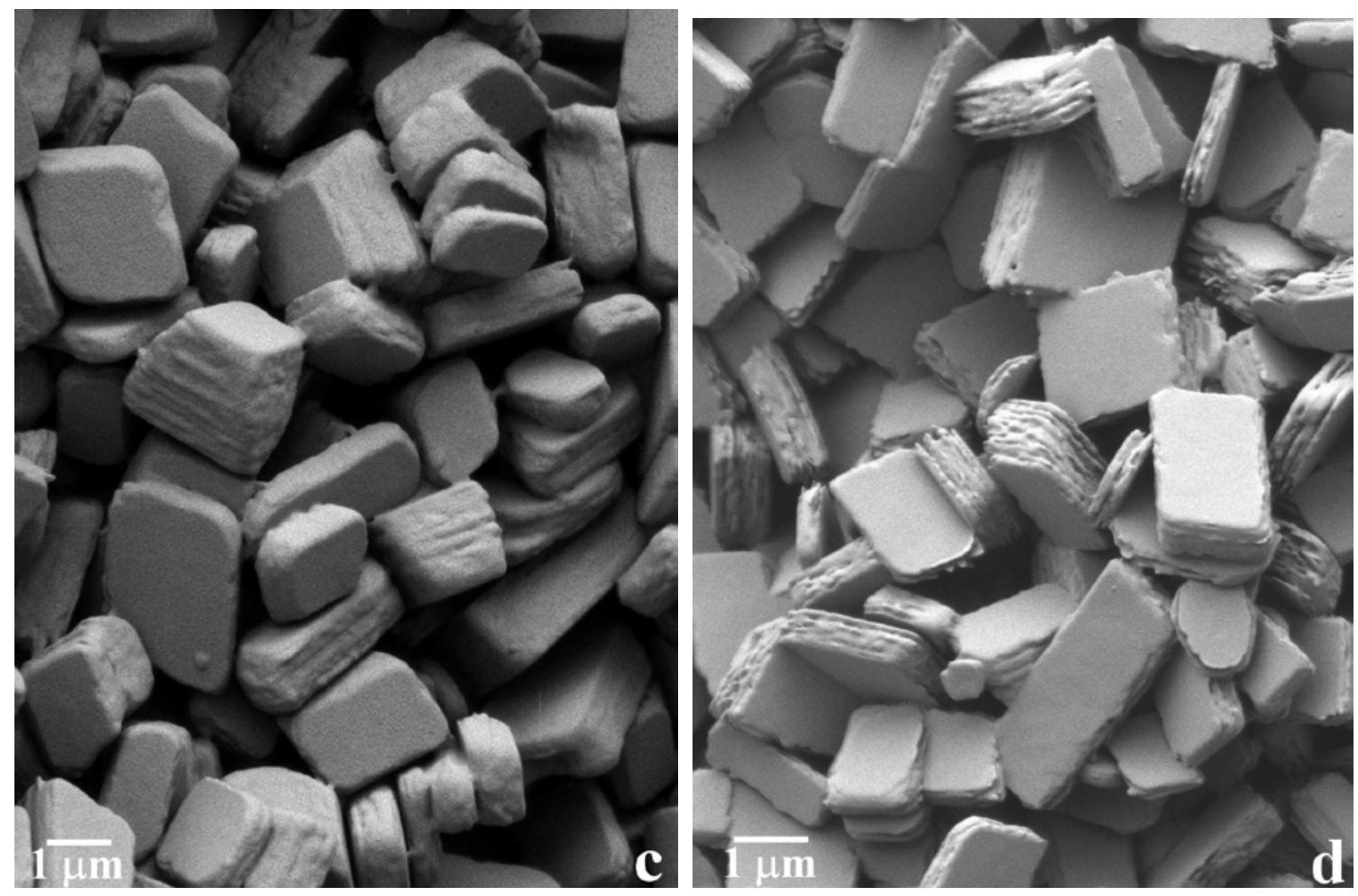

Fig.3. Scanning electron micrographs of: (a) cholesterol particles prepared by the rapid addition of $15 \mathrm{~cm}^{3}$ of water to the solution containing $20 \mathrm{mg}$ of cholesterol dissolved in $10 \mathrm{~cm}^{3}$ of 1-propanol at room temperature, aged for 10 min; (b) the same system as in (a), but aged for a month; (c) the same system as in (a), but aged for 2 days at $34{ }^{\circ} \mathrm{C}$; (d) the same system as in (a), but aged for $2 \mathrm{~h}$ in the presence of $0.4 \mathrm{M}$ sodium chloride.

The presence of water molecules in the suspension medium presumably induces 3-hydroxyl groups to protrude crystal surfaces, whereby their partial deprotonation causes the particles to be negatively charged at physiological $\mathrm{pH}$. This observation is consistent with the fact that particles in natural sols are most frequently dispersed on the anionic side. In general, hydrophobic surfaces in contact with aqueous media are more often negatively charged than positively charged, which is a consequence of the fact that cations (i.e., protons in case of water) are usually more hydrated than anions and, therefore, possess a greater tendency to reside in the bulk aqueous medium, whereas less 
hydrated and more polarizing anions (i.e., hydroxyl groups in case of water) have a greater tendency to be specifically adsorbed ${ }^{10}$. Furthermore, blood and lymph are comparatively dense and viscous dispersions that are kept in flowing, non-gelled state by preserving electronegative surface charge of blood cells, macromolecules and nutrients transferred therewith. Because carboxyl group tends to ionize at $\mathrm{pH}$ values above approximately 4, proteins are normally negatively charged in the $\mathrm{pH}$ range between 5 and 10, and become electropositive at around $\mathrm{pH} 2$ due to protonation of the amino group, with an isoelectric point at $\mathrm{pH}$ between 2 and 5 . Zeta potential of cholesterol particles in the dispersed form was $-80 \mathrm{mV}$ at this $\mathrm{pH}$, which falls in the surface charge range that promotes conditions for an exceptional stability of the corresponding suspensions. Zeta potential reaches $-14 \mathrm{mV}$ at $\mathrm{pH} 3.5$ and the isoelectric point in the vicinity of $\mathrm{pH} 2$, below which the particles become positively charged, presumably as the consequence of protonation of the surface hydroxyl groups. Crystallographic ordering of cholesterol, furthermore, points to hydroxyl groups as predominantly protruding the sides of the brick-shaped crystals (Fig.1), which accounts for the observed "face-to-face" aggregation of cholesterol particles. Whereas well-defined cholesterol platelets were produced within $\mathrm{pH}$ conditions that corresponded to either positively or negatively charged particle surfaces, precipitation at the isoelectric point due to reduced surface charge repulsion resulted in the formation of mutually stacked platelets. A further confirmation of the importance of electrostatic effects in the control of stability of cholesterol dispersions came from the experiments in which increased rates of aggregation of cholesterol platelets were observed in the presence of electrolytes (Fig.3d). Surface charge density of colloidal particles is directly proportional to the dielectric constant of the dispersion 
medium, which implies that increased ionic strength induces shrinkage of the charged layers around the dispersed particles and promotes conditions for their aggregation. Surface charge effects and their dependence on ionic strength have been previously hypothesized as crucially involved in the undesired "salting-out" precipitation of biological compounds that causes various pathological states of the cardiovascular system ${ }^{11}$. Also, in accordance with the Schulze-Hardy rule and the hydrophobic nature of cholesterol particles, the addition of electrolytes in the dispersion medium increased the rate of aggregation of individual platelets during the aging treatment in a direct proportion with concentration and valence of the introduced ionic species. The relationship between atherosclerotic deposits and high blood pressure (i.e. increased salt content) has thus been established for the first time at the level of basic physical interactions between colloidal cholesterol crystallites.

\section{Conclusion}

Surface charge effects can be from the discoursed results evidenced as crucially involved in stabilization of cholesterol dispersions. $\mathrm{pH}$ conditions that correspond to the isoelectric point of the dispersed particles have promoted increased aggregation rates, whereas the ones corresponding to negatively or positively charged particles induced preservation of their stability. And most importantly, increased ionic strengths have been shown to lead to increased rates of aggregation of the suspended cholesterol particles, indicating a direct link between the formation of pathological atherosclerotic deposits and high blood pressure, whereas the latter is known to be proportional to concentration of 
electrolytes in diet and blood. On the other hand, it is worth noting that this study has dealt with cholesterol particles dispersed in extremely simple environments comparing to the biological ones. For example, atherosclerotic plaque forms through a cooperative deposition of cholesterol, cholesteryl esters and triglycerides, mineral components (such as hydroxyapatite and other calcium phosphates), and various other macromolecules and accumulated cells, and that in liquid media rich in numerous molecular species that may affect the coagulation process in synergistic and unknown ways. Cholesterol is insoluble in blood and is transferred through the bloodstream by the action of lipoprotein complexes, the involvement of which in pathological deposition of cholesterol should not be underestimated as well. In the end, the whole popular dichotomy between "good" and "bad" cholesterol presents a misnomer, for there is only a single molecular form of cholesterol and what makes it "good" or "bad" is determined by whether it is high-density or low-density lipoprotein complexes that carry it through the bloodstream, respectively. And finally, atherosclerotic precipitation of cholesterol occurs in a dynamic environment permeated with a constant vascular flow, nowhere like the one existing in an ordinary solution. That this effect is of critical importance is best illustrated by the finding that atherosclerotic plaque forms at sites associated with disturbed blood flow patterns, such as along vessel curvatures and bifurcations ${ }^{12}$. Nevertheless, the author believes that simple ways to produce uniform and well-defined cholesterol crystals and the evidenced significance of charge effects in their aggregation might provide a convenient model for further studies and a novel direction in the biochemical manipulation of in vivo behavior of cholesterol precipitates and medical prevention of their long-term deposition. 


\section{References:}

${ }^{1}$ F. R. Maxfield, I. Tabas - "Role of Cholesterol and Lipid Organization in Disease”, Nature 438, 612 - 21 (2005).

${ }^{2}$ B. M. Craven - "Crystal Structure of Cholesterol Monohydrate”, Nature 260, 727 - 9 (1976).

${ }^{3}$ H. S. Shieh, L. G. Hoard, C. E. Nordman - "Crystal Structure of Anhydrous Cholesterol”, Nature 267, 287 - 9 (1977).

${ }^{4}$ R. S. Abendan, J. A. Swift - "Surface Characterization of Cholesterol Monohydrate Single Crystals by Chemical Force Microscopy”, Langmuir 18, 4847 - 53 (2002).

${ }^{5}$ R. S. Abendan, J. A. Swift - "Dissolution on Cholesterol Monohydrate Single-Crystal Surfaces Monitored by in Situ Atomic Force Microscopy”, Crystal Growth \& Design 5, 2146 - 53 (2005).

${ }^{6}$ V. Uskoković, E. Matijević - "Uniform Particles of Pure and Silica Coated Cholesterol”, Journal of Colloid and Interface Science 315 (2) 500 - 511 (2007).

7 V. Uskoković - "Insights into Morphological Nature of Precipitation of Cholesterol", Steriods DOI:10.1016/j.steroids.2007.12.005 (2008).

${ }^{8}$ V. Uskoković - "Composites Comprising Cholesterol and Carboxymethyl Cellulose", Colloids and Surfaces B: Biointerfaces 61, 250 - 261 (2008).

${ }^{9}$ V. Uskoković - "Morphological Study of Emulsion-Assisted Cholesterol Precipitation Processes", Molecular Crystals and Liquid Crystals 474, 77 - 88 (2007).

${ }^{10}$ D. J. Shaw - "Introduction to Colloid and Surface Chemistry", Butterworth Heinemann, Oxford, UK (1992).

${ }^{11}$ T. M. Riddick - "Control of Colloid Stability through Zeta Potential and its Relationship to Cardiovascular Disease”, Livingston Publishing, Wynnewood, PA (1968).

${ }^{12}$ P. A. VanderLaan, C. A. Reardon, G. S. Getz - "Site Specificity of Atherosclerosis: Site-Selective Responses to Atherosclerotic Modulators", Arteriosclerosis, Thrombosis, and Vascular Biology 24, 12 - 22 (2004). 